\title{
Spin Injection into a Luttinger Liquid
}

\author{
Qimiao Si \\ Department of Physics, Rice University, Houston, TX 77251-1892
}

\begin{abstract}
We study the effect of spin injection into a Luttinger liquid. The spin-injection-detection setup of Johnson and Silsbee is considered; here spins injected into the Luttinger liquid induce, across an interface with a ferromagnetic metal, either a spin-dependent current $\left(I_{s}\right)$ or a spin-dependent boundary voltage $\left(V_{s}\right)$. We find that the spin-charge separation nature of the Luttinger liquid affects $I_{s}$ and $V_{s}$ in a very different fashion. In particular, in the Ohmic regime, $V_{s}$ depends on the spin transport properties of the Luttinger liquid in essentially the same way as it would in the case of a Fermi liquid. The implications of our results for the spin-injection-detection experiments in the high $T_{c}$ cuprates are discussed.
\end{abstract}

PACS numbers: 71.10.Hf, 73.40. -c, 71.27. +a, 72.15.Gd

Spin-charge separation has long been proposed to describe the normal state of the high $T_{c}$ cuprate superconductors [1]. Existing experimental results cited as evidences for spin-charge separation are mostly on transport properties [2]. Since only charge transport properties have so far been measured, the inference about the coupling, or lack thereof, between the underlying spin and charge excitations is indirect. It would appear natural that spin transport, when combined with charge transport, should be useful in this context. Indeed we have recently proposed to probe spin-charge separation using a comparison between the temperature dependence of the yet-to-be-measured spin resistivity and that of the known electrical resistivity [3]. Several factors point to the feasibility of experimentally measuring spin transport in the cuprates using the spin-injection-detection technique [ [- 6 . First of all, progresses in the preparation of the manganite-cuprate heterostructures appear to have led to the first demonstration of spin injection into the cuprates (albeit in the superconducting state) [7],8]. Secondly, the spin-diffusion length in the cuprates has been estimated to fall in the range required by this technique [3].

In light of the new experiments on the high $T_{c}$ cuprates, it is important to understand how spin injection into a non-Fermi liquid differs from spin injection into a Fermi liquid. The effects of spins injected into a non-interacting electron system has been studied in the past by Johnson and Silsbee and others 任 6,9,10, following the initial proposals for spin injection and detection 11, 12].

Here we address the influence of spin-charge separation in the bulk metal on the boundary voltage/current measured in a spin-injection experiment. For definiteness, the one-dimensional Luttinger liquid [13] is used as a prototype for a spin-charge-separated metal. Crucial for our analysis is the fact that, the interface transport involves the binding of spinons and holons which then tunnel as a whole from the Luttinger liquid into the ferromagnetic metal. We find that the spin-charge-separation nature of the Luttinger liquid affects the boundary voltage and boundary current in a very different fashion.

Figs. 1 1a) and 1b) illustrate two specific geometries involving a single channel Luttinger liquid (LL). The Luttinger liquid is in contacts with two itinerant ferromagnets, FM1 and FM2. The magnetization of FM1 is chosen as the $\uparrow$ direction. The magnetization of FM2 is either parallel $(\sigma=\uparrow)$ or antiparallel $(\sigma=\downarrow)$ to that of FM1. One passes an electrical current $(I)$ across the FM1-LL interface. This current serves to inject non-equilibrium magnetization into the Luttinger liquid. For a given $\sigma$, $I_{\sigma}$ represents the induced current across the LL-FM2 interface in a closed circuit and $V_{\sigma}$ is the induced boundary voltage $\left(V_{\sigma}\right)$ in an open circuit. The spin-dependent current, $I_{s}$, is defined as the difference between the induced current when the magnetizations of the two ferromagnets are in parallel and that when they are antiparallel. Likewise, the spin-dependent voltage, $V_{s}$, is the difference between the induced boundary voltages in the corresponding two cases. The setups illustrated in Figs. 1 1a) and 1b) differ in two regards. First of all, while the Luttinger liquid is in point contacts with both of the ferromagnets in Fig. 17a), in Fig. 17b) it is in contact with FM2 over an extended spatial region. Secondly, unlike in Fig. 1 1a) the injection and detection loops in Fig. 1 $1 \mathrm{~b}$ ) are closed through contacts with LL far away both FM1 and FM2. The setup of Fig. 1b) is perhaps easier to implement experimentally. On the other hand, that of Fig. 1a) is easier to analyze theoretically. To illustrate the basic principle, in the rest of this paper we will focus on Fig. 11a).

The Hamiltonian of the Luttinger liquid can be written as

$$
\begin{aligned}
H_{l u t} & =H_{\rho}+H_{s}+H^{\prime} \\
H_{\rho} & =\frac{1}{2 \pi} v_{\rho} \int d x\left[K_{\rho}\left(\pi \Pi_{\rho}\right)^{2}+\frac{1}{K_{\rho}}\left(\partial_{x} \phi_{\rho}\right)^{2}\right] \\
H_{s} & =\frac{1}{2 \pi} v_{s} \int d x\left[K_{s}\left(\pi \Pi_{s}\right)^{2}+\frac{1}{K_{s}}\left(\partial_{x} \phi_{s}\right)^{2}\right]
\end{aligned}
$$

where $H_{\rho}$ and $H_{s}$ are respectively the Hamiltonian for the charge $(\rho)$ and spin $(s)$ bosons, $\phi_{\rho}$ and $\phi_{s} ; \Pi_{\rho}$ and $\Pi_{s}$ 


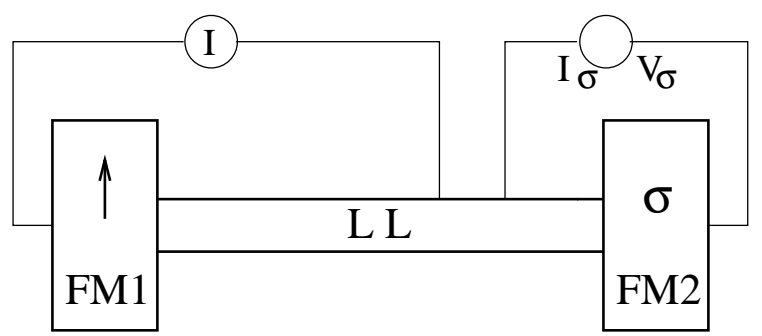

a)

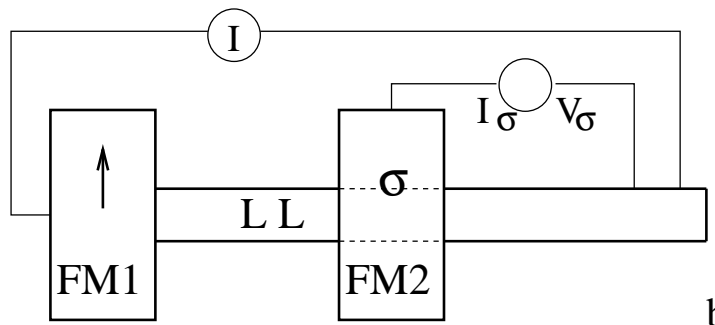

b)

FIG. 1. Two possible set ups for spin-injection-detection experiment on a one-channel Luttinger liquid (LL). The magnetization of the ferromagnetic metal FM2 is either parallel $(\sigma=\uparrow)$ or antiparallel $(\sigma=\downarrow)$ to that of the ferromagnetic metal FM1. $I$ is the injection current. $I_{\sigma}\left(V_{\sigma}\right)$ is the current (boundary voltage) induced across the LL-FM2 interface when the circuit is closed (open). The two interfaces in a) are separated by a distance $d$.

are the corresponding conjugate momenta. The charge and spin velocities, $v_{\rho}$ and $v_{s}$, and Luttinger liquid parameters, $K_{\rho}$ and $K_{s}$, are determined by the forward scattering interactions. We consider the case when the spin-rotational invariance is preserved so that $K_{s}=1$.

We focus on the regime where spin transport inside the Luttinger liquid is diffusive, with a spin diffusion constant $D_{s}$. The diffusive transport is the result of the dissipative terms in the Hamiltonian, $H^{\prime}$, which also lead to a finite spin relaxation time $T_{1}$. The precise form of $H^{\prime}$ is however unimportant for our purpose in this paper and is left unspecified here. (It will, of course, determine the specific temperature dependences of $D_{s}$ and $T_{1}$.) To simplify the discussion, we assume that the FMs are halfmetallic ferromagnets. We will also neglect the electron interactions inside the ferromagnets [14]. The free electron Hamiltonians for FM1 and FM2 are respectively

$$
H_{1}=\sum_{k} \epsilon_{k}^{1} c_{k \uparrow}^{\dagger} c_{k \uparrow}
$$

and

$$
H_{2}=\sum_{k} \epsilon_{k}^{2} c_{k, \sigma}^{\dagger} c_{k, \sigma}
$$

where $\epsilon_{k}^{1}$ and $\epsilon_{k}^{2}$ are the corresponding energy dispersions.

Since we will consider only the cases when the magnetizations of the two ferromagnets are either parallel or anti-parallel with each other, we need only the kinetic equation for the longitudinal component of the magnetization. We introduce $m(x)$ to denote the deviation of the steady state magnetization density from the corresponding equilibrium value. The FM1-LL and LL-FM2 interfaces are located at $x=0$ and $x=d$, respectively. The non-equilibrium magnetization density $m(x)$ satisfies the following [15]

$$
-D_{s} \frac{\partial^{2} m}{\partial x^{2}}=-\frac{m(x)}{T_{1}}
$$

where $T_{1}$ is the longitudinal spin relaxation time. The boundary conditions will depend on the details of the interface. Leaving more general cases for elsewhere [16], we assume that no spin-flip scattering exists at the interface. In this case, the spin current $j_{s}$ is conserved across the interface. Given that $j_{s}\left(x=0^{-}\right)=\mu_{B} I / e$, where $\mu_{B}$ is the Bohr magneton and $e$ the electron charge, the boundary condition at the FM1-LL interface is,

$$
-D_{s} \partial m /\left.\partial x\right|_{x=0}=\mu_{B} I / e
$$

Likewise, at the LL-FM2 interface,

$$
-D_{s} \partial m /\left.\partial x\right|_{x=d}=\mu_{B} I_{\sigma} / e
$$

The induced current $I_{\sigma}$ depends on the drop of the nonequilibrium magnetization across the LL-FM2 interface, which is equal to $\Delta m=m(d)$. We separately discuss the closed circuit and open circuit cases.

Consider, first, the case of a closed circuit. The drop of non-equilibrium magnetization, $\Delta m$, leads to a drop in the effective magnetic field,

$$
\Delta H=\Delta m / \chi
$$

across the LL-FM2 interface. Here, $\chi$ is the uniform spin susceptibility of the Luttinger liquid. $\Delta H$ provides a driving force for spinons to move across the interface. Since only electrons can tunnel across the barrier, spinons bind with holons and move across the interface as a whole. A finite electrical current, $I_{\sigma}^{\text {closed }}$, is then induced by $\Delta H$. To calculate $I_{\sigma}^{\text {closed }}$, we follow the general procedure of Kane and Fisher [17] and integrate out all the degrees of freedom of the Luttinger liquid except at the site of contact, $x=d$. This leads to an effective action entirely determined by the boson field $\phi_{\rho} \equiv \phi_{\rho}(d)$ and $\phi_{s} \equiv \phi_{s}(d)$ :

$$
S_{\text {site }}=K_{\rho} \frac{1}{\beta} \sum_{\omega_{n}}\left|\omega _ { n } \left\|\left.\phi_{\rho}\left(\omega_{n}\right)\right|^{2}+\frac{1}{\beta} \sum_{\omega_{n}}\left|\omega_{n} \| \phi_{s}\left(\omega_{n}\right)\right|^{2}\right.\right.
$$

where $\beta$ is the inverse of temperature and $\omega_{n}$ the bosonic Matsubara frequencies. In deriving this on-site action, we have neglected the effect of the non-equilibrium magnetization, $m(x)$. This is appropriate for the Ohmic regime $k_{B} T>\mu_{B} m / \chi$. We are now faced with a problem of one 


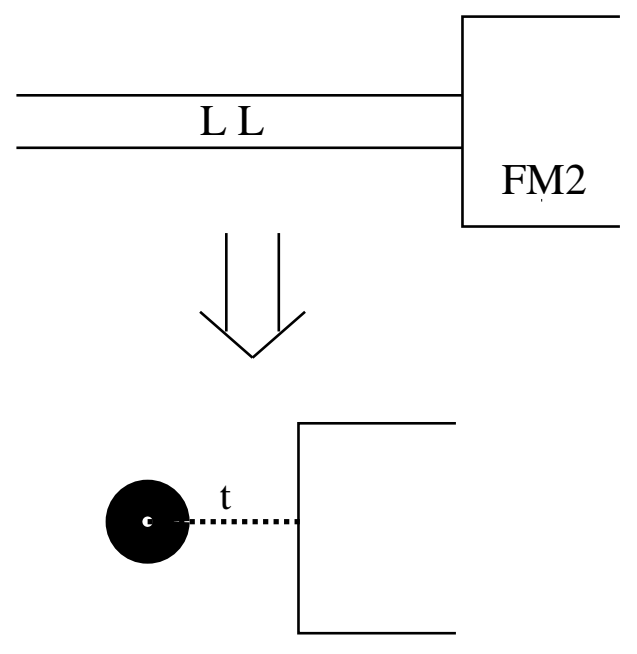

FIG. 2. Reduction of the coupled LL-FM2 problem to a retarded impurity, described by the action given in Eq. (8), coupled to a three-dimensional ferromagnetic metal. The coupling is through the tunneling matrix $t$.

[18] retarded impurity - whose dynamics is controlled by Eq. (8) - coupled to a three dimensional ferromagnetic metal. This effective impurity problem is illustrated in Fig. 2. The impurity problem can also be written in a Hamiltonian form, by introducing a fictitious bosonic bath.

From the one-particle tunneling Hamiltonian, one can construct respectively the charge current operator $J$ and spin current operator $J_{M}$. They are as follows,

$$
\begin{aligned}
J / e= & J_{M} / \mu_{B} \\
= & \frac{t}{\sqrt{2 \pi a}}\left[F_{\sigma-}^{\dagger} \mathrm{e}^{-i \frac{\phi_{\rho}+\theta_{\rho}}{\sqrt{2}}-i \sigma \frac{\phi_{s}+\theta_{s}}{\sqrt{2}}}\right. \\
& \left.+F_{\sigma+}^{\dagger} \mathrm{e}^{-i \frac{-\phi_{\rho}+\theta_{\rho}}{\sqrt{2}}-i \sigma \frac{-\phi_{s}+\theta_{s}}{\sqrt{2}}}\right] c_{\sigma}-\text { H.c. }
\end{aligned}
$$

where $F_{\sigma \pm}^{\dagger}$ are the Klein operators for the left and right moving branches and $a$ is a lattice cutoff [13,19]. Here, $c_{\sigma}$ is the annihilation operator for the Wannier orbital of the conduction electrons at the contact, and $t$ is the tunneling matrix. That the interface charge and spin current operators are simply related to each other 20 in spite of the separated spin and charge excitations in the bulk Luttinger liquid reflects the simple physics that, only a bare electron tunnels across the interface.

We can now calculate $I_{\sigma}^{\text {closed }}$ in the Ohmic regime using the Kubo formalism,

$$
I_{\sigma}^{\text {closed }}=(-\Delta H) \lim _{\omega \rightarrow 0} \frac{-\operatorname{Im} \pi_{J J_{M}}\left(\omega+i 0^{+}\right)}{\omega}
$$

where $\pi_{J J_{M}}$ is the charge current-spin current correlation function. Similar to the Kane-Fisher problem, for repulsive interactions $K_{\rho}<1$, the tunneling term is an irrelevant coupling in the renormalization group sense. We can then calculate $\pi_{J_{J}}$ perturbatively in $t$. The result is as follows,

$$
I_{\sigma}^{\text {closed }}=C\left(k_{B} T\right)^{1 / 2 K_{\rho}-1 / 2} e \mu_{B} \Delta m / \chi
$$

where $C=c N_{F} t^{2} / W^{1 / 2 K_{\rho}+1 / 2}$. Here $N_{F}$ is the density of states of FM2 at the Fermi energy, $W$ is a typical bare energy scale associated with the electrons in the Luttinger liquid, and $c$ is a constant of order unity. The induced spin-dependent current across the LL-FM2 interface, $I_{s}=I_{\uparrow}^{\text {closed }}-I_{\downarrow}^{\text {closed }}$ can now be determined, from Eqs. (4.5) 6, 11). The result is as follows,

$$
I_{s} / I=C\left(k_{B} T\right)^{1 / 2 K_{\rho}-1 / 2} \frac{\mu_{B}^{2}}{\chi} \frac{T_{1}}{\delta_{s} \sinh \left(d / \delta_{s}\right)}
$$

where $\delta_{s}=\sqrt{D_{s} T_{1}}$ is the spin-diffusion length of the Luttinger liquid.

We now turn to the open circuit case. Here, in order to balance the current induced by the magnetization drop, a boundary voltage, $V_{\sigma}$, develops across the LL-FM2 interface. The induced current in this case is

$$
\begin{aligned}
I_{\sigma}^{\text {open }}= & (-\Delta H) \lim _{\omega \rightarrow 0} \frac{-\operatorname{Im} \pi_{J J_{M}}\left(\omega+i 0^{+}\right)}{\omega} \\
& +V_{\sigma} \lim _{\omega \rightarrow 0} \frac{-\operatorname{Im} \pi_{J J}\left(\omega+i 0^{+}\right)}{\omega}
\end{aligned}
$$

which leads to

$$
I_{\sigma}^{\text {open }}=C\left(k_{B} T\right)^{1 / 2 K_{\rho}-1 / 2} e\left(\mu_{B} \Delta m / \chi+e V_{\sigma}\right)
$$

Setting $I_{\sigma}^{\text {open }}=0$ in Eq. (13), and combining with Eqs. (4.5.,6), we arrive at the following expression for the spindependent boundary voltage, $V_{s}=V_{\uparrow}-V_{\downarrow}$ :

$$
V_{s} / I=\frac{\mu_{B}^{2}}{e^{2} \chi} \frac{T_{1}}{\delta_{s} \sinh \left(d / \delta_{s}\right)}
$$

Eqs. (12,15) are the main results of this work. Several comments are in order.

First of all, it is instructive to see how our results reduce to those for free electrons [4 6, 9, 10] when electron interactions are reduced to zero. For non-interacting electrons, spin diffusion $D_{s}$ is reduced to the usual electron diffusion constant $D=v_{F}^{2} \tau$, where $v_{F}$ is the Fermi velocity and $\tau$ the transport scattering time. In addition, $\chi=\mu_{B}^{2} N_{F}^{P}$, where $N_{F}^{P}$ is the density of states at the Fermi energy. Straightforward manipulation leads to $V_{s} / I=\rho \delta_{s}^{0} / \sinh \left(d / \delta_{s}^{0}\right)$, where $\rho$ is the electrical resistivity of the bulk metal and $\delta_{s}^{0}=\sqrt{D T_{1}}$. In addition, for non-interacting electrons, $K_{\rho}=1$, and our expression for $I_{s}$ is reduced to $I_{s} / I=\left(e^{2} N_{F} N_{F}^{P} t^{2}\right) \rho \delta_{s} / \sinh \left(d / \delta_{s}\right)$.

Secondly, we note that for the Luttinger liquid, the temperature dependence of $I_{s}$ is not solely determined by that of the bulk spin diffusion and relaxation properties of 
the Luttinger liquid. There is an additional temperaturedependent factor, with a power which explicitly depends on the Luttinger liquid parameter $K_{\rho}$. This additional temperature-dependent factor, however, cancels out in $V_{s}$. This last result can ultimately be traced to the fact that the interface charge current and spin current are directly related to each other, in spite of the spin-charge separation nature of the bulk Luttinger liquid. On this ground, we expect the expression for $V_{s}$ to be valid very generally, so long as one particle processes dominate the interface transport and no strong-coupling (in $t$ ) phenomena 21] take place. The boundary voltage $V_{s}$ is hence more useful than $I_{s}$ for the purpose of extracting bulk spin transport properties of strongly correlated metals, including the high $T_{c}$ cuprates.

The general expression for the spin-dependent boundary voltage suggests the following procedure to measure the temperature dependence of the spin transport scattering rate, $1 / \tau_{t r, s p i n}$, of correlated metals. The latter is the quantity of interest in probing spin-charge separation [3]. In strongly interacting metals, it is likely that the dissipations for both the spin current and total spin come primarily from electron-electron interactions. The spin relaxation time and spin transport relaxation rate are then proportional to each other: $\frac{1}{T_{1}} \approx\left(\lambda_{s o}\right)^{2} \frac{1}{\tau_{t r, s p i n}}$, where $\lambda_{\text {so }}$ is the dimensionless spin-orbit coupling constant. When the sample thickness $d$ is small compared to the spin diffusion length $\delta_{s}$, the temperature dependence of $\chi V_{s} / I$ is then directly proportional to the temperature dependence of $\tau_{t r, s p i n}$. On the other hand, for thickness much larger than the spin diffusion length, the temperature dependence of $\ln \left(\chi V_{s} / I\right)$ is directly proportional to that of $1 / \tau_{\text {tr,spin. }}$. This procedure does not require measurement in a series of samples of different thicknesses as was necessary in the case of simple metals [5] - and may be experimentally easier to implement.

To summarize, we have studied the effects of spin injection into a Luttinger liquid. Our conclusion that the temperature dependence of the boundary voltage depends on the bulk spin-transport properties only is expected to be generally applicable and of direct relevance to the spininjection-detection experiments in the cuprates.

I would like to thank N. Andrei, M. Johnson, Q. Li and A. J. Rimberg for useful discussions, and the Aspen Center for Physics and the CM/T group at NHMFL/FSU for hospitality and support during different stages of this work. The work has also been supported by NSF Grant No. DMR-9712626, a Robert A. Welch Foundation grant and an A. P. Sloan Fellowship.

[1] P. W. Anderson, Science 235, 1196 (1987); Phys. Rev.
Lett. 64, 1839 (1990).

[2] P. W. Anderson, The Theory of Superconductivity in the High- $T_{c}$ Cuprates (Princeton University Press, Princeton, NJ 1997).

[3] Q. Si, Phys. Rev. Lett. 78, 1767 (1997).

[4] M. Johnson and R. H. Silsbee, Phys. Rev. Lett. 55, 1790 (1985).

[5] M. Johnson, Phys. Rev. Lett. 70, 2142 (1993) and references therein.

[6] M. Johnson and R. H. Silsbee, Phys. Rev. B 37, 5312 (1988); ibid. B 35, 4959 (1987).

[7] V. A. Vas'ko, V. A. Larkin, P. A. Kraus, K. R. Nikolaev, D. E. Grupp, C. A. Nordman, and A. M. Goldman, Phys. Rev. Lett. 78, 1134 (1997).

[8] Z. W. Dong, R. Ramesh, T. Venkatesan, M. Johnson, Z. Y. Chen, S. P. Pai, V. Talyansky, R. P. Sharma, R. Shreekala, C. J. Lobb and R. L. Greene, Appl. Phys. Lett. 71, 1718 (1997).

[9] A. Fert and S.-F. Lee, Phys. Rev. B53, 6554 (1996).

[10] S. Hershfield and H. L. Zhao, Phys. Rev. B56, 3296 (1997).

[11] A. G. Aronov, JETP Lett. 24, 32 (1976).

[12] R. H. Silsbee, Bull. Magn. Res. 2, 284 (1980).

[13] For a review see J. Voit, Rep. Prog. Phys. 58, 977 (1995).

[14] Having super-ohmic spectral functions, the spin waves in the ferromagnets are unimportant for our analysis.

[15] The kinetic equation for the transverse magnetization would be more complex, due to the Leggett-Rice effect. A. J. Leggett and M. J. Rice, Phys. Rev. Lett. 20, 586 (1968); A. J. Leggett, J. Phys. C3, 448 (1970).

[16] Q. Si, unpublished.

[17] C. L. Kane and M. P. A. Fisher, Phys. Rev. Lett. 68, 1220 (1992); Phys. Rev. B46, 15233 (1992).

[18] With more than one transverse channels, the effective problem describes multiple, spatially separated, impurities coupled to a three dimensional metal. N. Andrei and Q. Si, unpublished.

[19] G. Kotliar and Q. Si, Phys. Rev. B53, 12373 (1996).

[20] When FM2 is not half-metallic, the relationship between $J$ and $J_{M}$ will be more complex. The conclusions are, however, essentially the same as give here 16 .

[21] See, e.g., N. P. Sandler, C. de C. Chamon, and E. Fradkin, Phys. Rev. B57, 12324 (1998) and references therein. 